\title{
Modelling fluid structure interaction using one-way coupling and proper orthogonal decomposition (POD)
}

\author{
S. Shinde \& M. Pandey \\ Indian Institute of Technology Madras, India
}

\begin{abstract}
Fluid structure interaction (FSI) problems are becoming highly complex as FSI has vast applications, which help to model many real life problems. Here, a oneway coupling strategy has been proposed for the FSI problem, where fluid has been modelled in computational fluid dynamics (CFD) and its interface with the solid structure has been modelled with a no-slip no-penetration boundary condition. Using Abaqus CFD simulations, the calculated interface pressure has been applied on the POD modes of the solid structure domain to obtain the displacement field. Here, we assumed that the motion of the flexible solid structure has no effects on the fluid flow as it is a one-way coupling. This assumption is well governed for the less flexible structure. However, it has been noted that this shows good predictions for flexible structures too. The benefit of this approach is that for the same interface boundary of the FSI domain, we can change the structural material properties and predict the results without going for the complete FSI simulation problem. Here, we solved a case study problem example for the fluid flow over a flexible beam and results are validated using the Abaqus FSI Cosimulation.
\end{abstract}

Keywords: FSI, POD, vortex shedding induced vibration, one-way coupling, reduced order modelling (ROM).

\section{Introduction}

As solving an FSI problem is computationally costly and time consuming, so many different approaches and trends have been discovered to reduce the computational cost. POD has been used more efficiently for the model order reduction of nonlinear structural dynamics problems (Hung and Senturia [1]). Even fluid flow 
problems have been also solved using a POD based ROM technique (Akthar et al. [2]). For fluid structure interaction problems various approaches have been considered, such as POD-FSM (POD-force survey method) by Liang and Dong [3], minimum residue projection method (MRP) by Tallet et al. [4] etc. Here, we propose the one-way coupling strategy using POD for the FSI problem.

This paper is structured as follows: section 2 contains the methodology, section 3 covers the problem definition, section 4 deals with the solution by the proposed approach, followed by section 5, which deals with the results and discussions.

\section{Methodology}

In this paper, we propose the decoupled modelling approach of the FSI problem. We model the fluid domain and structure domain separately in Abaqus. The structure domain in the fluid is modelled as a rigid cavity by means of no-slip nopenetration boundary condition at the interface. Then, the fluid problem has been solved using Abaqus CFD simulation for the pressure variation $P(X, t)$ at the FSI interface.

The structure has been modelled separately and the POD modes were found using the singular value decomposition (SVD) technique by snapshot method. This part will be discussed in detail in the following sections. The generated POD ROM parameters i.e. mass matrix $(\mathrm{M})$ and stiffness matrix $(\mathrm{K})$ are obtained as per the Euler Bernoulli beam equation for the current problem definition. These ROM parameters govern the dynamics of the system. The pressure force will be applied on the corresponding POD mode of the structure and the displacement field will be calculated by solving the Euler Bernoulli beam equation over the domain. In section 3, we discuss the example case study problem and computational domain in detail.

\section{Problem definition}

The case study problem geometry along with the boundary conditions is as shown in fig. 1. This model is inspired from the vortex shedding induced energy harvesting model described in Weinstein et al. [5]. As the fluid flows over a rigid cylindrical bluff body with sufficiently high Reynolds number results in the vortex shedding effect in the wake region. So when we put the flexible structure in the wake region it will start vibrating because of the differential fluid pressure across the cantilevered beam.

The fluid is modelled as 2D in Abaqus and the beam structure is modelled as a $3 \mathrm{D}$ beam. The fluid domain is discretised in such a way that the Courant number is less than 0.5 . The grid independence and domain independence study has been performed on the fluid mesh. The fluid domain has the rigid cylindrical bluff body of diameter $2.5 \mathrm{~mm}$. The beam structure has a length of $25 \mathrm{~mm}$, a width of $5 \mathrm{~mm}$ and a thickness of $0.4 \mathrm{~mm}$. The beam is modelled in such way that the vortex shedding frequency of the fluid will match approximately with the first natural 
frequency of the beam in order to have resonance and maximum vibration which allows us to harness the maximum available energy using piezoelectricity.

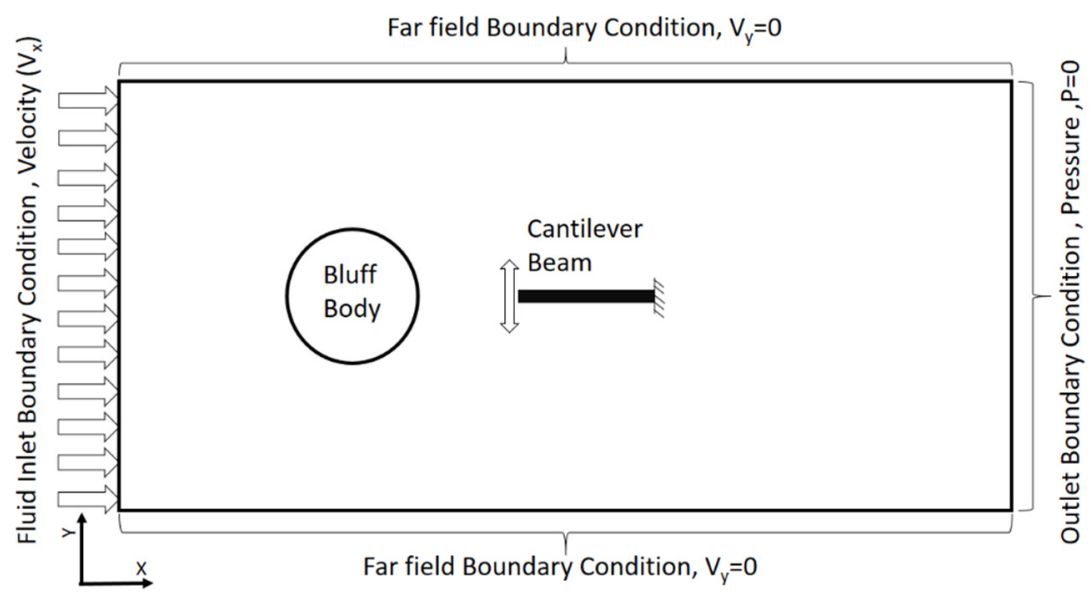

Figure 1: Case study problem geometry with boundary conditions.

Here, for such type of application it is important to know how the change in material parameters of the beam structure will affect the dynamics of the structure. Here, for the current application air is considered as a fluid with density $\left(\rho_{\text {air }}\right)$ $1.225 \mathrm{~kg} / \mathrm{m}^{3}$ and viscosity $\left(\mu_{\text {air }}\right) 1.983 \times 10^{-5} \mathrm{~Pa} . \mathrm{s}$. For the beam, the material properties are assumed as density $\left(\rho_{s}\right) 7800 \mathrm{~kg} / \mathrm{m}^{3}$, Young's modulus (E) $5 \times 10^{8} \mathrm{~Pa}$ and Poison's ratio as 0.3 .

\section{Proposed approach: one-way coupling}

Fluid and structure have been modelled separately in a computational domain using Abaqus. However, to validate the results of one-way coupling based on POD based calculations, we have used FSI co-simulation of Abaqus.

\subsection{Fluid domain}

Fluid domain is modelled as 2D in Abacus (as shown in fig. 1), the inlet velocity boundary condition, far field boundary condition and outlet boundary condition have been specified. The cylindrical bluff body and the beam both are modelled as the rigid cavity in the fluid domain by means of no slip, no penetration boundary at the interface. The fluid domain has dimensions of $200 * 400 \mathrm{~mm}$ based on the domain independence study performed. The cylindrical bluff body and beam is located at the center in y-direction. The center of the cylindrical bluff body and the fixed end of the cantilever beam is located at $100 \mathrm{~mm}$ and $160 \mathrm{~mm}$ from the left end of the domain respectively (as shown in fig. 1). The computational fluid mesh domain is as shown in fig. 2 . 


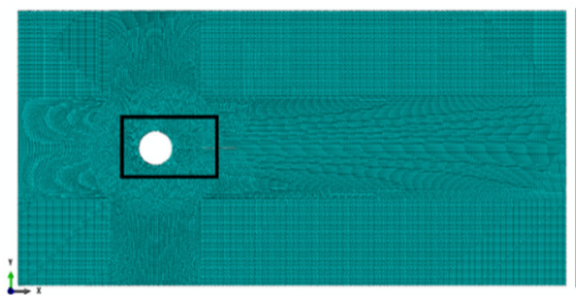

(A)

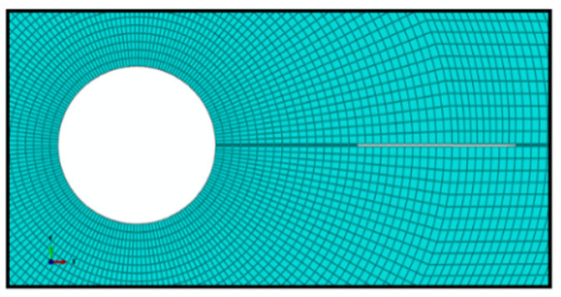

(B)

Figure 2: $\quad$ Full computational fluid domain mesh (A), zoomed mesh with beam and cylindrical bluff body modelled as no-slip no-penetration boundary condition $(\mathrm{B})$.

The simple fluid CFD problem has been solved using the Abaqus CFD for the interface pressure on the beam which is analytically governed by the NavierStokes eqn. (1) and continuity eqn. (2) for the incompressible flow. They can be as follows (referred from [6])

$$
\begin{gathered}
\frac{\partial u_{i}}{\partial t}+\frac{\partial}{\partial x_{j}}\left(u_{i} u_{j}\right)=\frac{1}{\rho} \frac{\partial \mathrm{p}}{\partial \mathrm{x}_{\mathrm{i}}}+v \frac{\partial^{2} u_{i}}{\partial x_{j} \partial x_{i}} \\
\frac{\partial u_{i}}{\partial x_{j}}=0
\end{gathered}
$$

where, $\mathrm{u}$ and $\mathrm{p}$ represent the velocity and pressure field respectively at time instant $t$, over the entire fluid domain. $\rho$ and $v$ represents the density and kinematic viscosity of the fluid and also $\mathrm{i}, \mathrm{j}=1,2.3$; Cartesian components along $\mathrm{x}, \mathrm{y}, \mathrm{z}-$ directions respectively.

The CFD simulation is performed and the pressure values at the fluid-solid interface has been found (the pressure contour plot for a time instant is as shown in fig. 3).

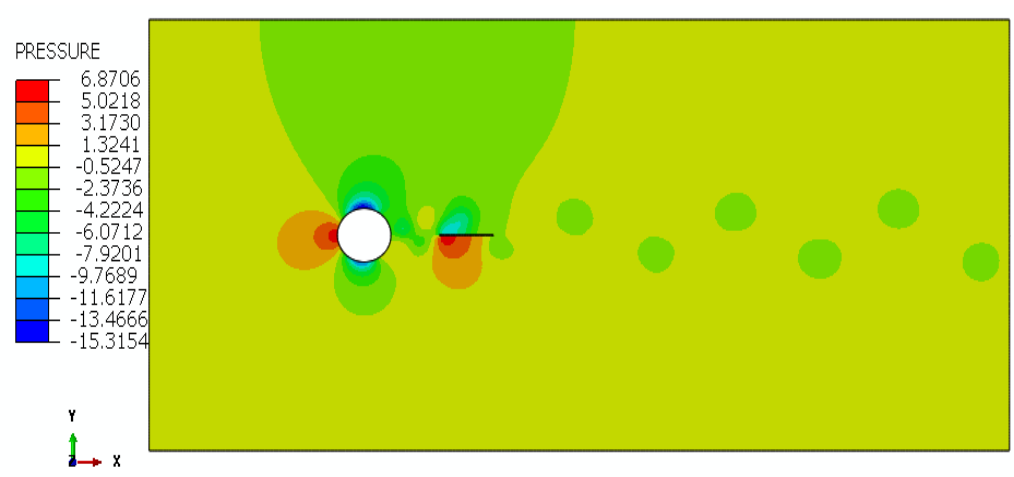

Figure 3: Pressure contour at a time instant over fluid domain. 
Let, $\mathrm{P}_{\text {top }}(\mathrm{X}, \mathrm{t})$ and $\mathrm{P}_{\text {bottom }}(\mathrm{X}, \mathrm{t})$ be the pressure variation above and below the beam, noted at each time instant $t$. Then the differential pressure on the top of the beam is given by eqn (3). Such approach has been previously applied in a similar problem by Huang [7].

$$
\delta p(X, t)=P_{\text {top }}(X, t)-P_{\text {bottom }}(X, t)
$$

\subsection{Structure domain}

It should be noted that the beam structure is modelled just for capturing the domain dynamics using the method of snapshots as mentioned by Hung and Senturia [1] using POD based reduced order modelling (ROM).

\subsubsection{Construction of POD based ROM}

For this purpose, the Euler beam has been modelled in Abaqus with the same dimension and material properties as mentioned in earlier sections. Then the simple FEA analysis has been performed for the periodically varying concentrated load at the free end of the cantilever beam and the displacement field at each node at equally spaced time intervals has been recorded in the snapshot matrix. The snapshot matrix has been constructed as each row of the matrix corresponds to the single node and each column of the matrix corresponds to the single time snapshot. So, the size of the snapshot matrix (W) will become the number of nodes $\times$ the number of time snapshots.

Then the global basis function is found by using singular value decomposition (SVD) as shown in eqn (4). Readers are encouraged to read the theory of SVD and its applications from the book [8]. Many commercially available software has the computationally efficient algorithm to calculate SVD.

$$
W=U S V^{T}
$$

Columns of $U$ will give the global POD basis functions for the displacement field. It can be plotted along the length of the beam as in fig. 4. From fig. 4, one may observe that the nonlinear POD modes have a similar pattern to that of the linear mode shapes of the beam, but it is important to note that the POD mode captures nonlinearity in the beam as well. The diagonal matrix $\mathrm{S}$ contains the singular values in a descending fashion which has significance on the amount of energy captured in that particular POD mode. Based on this study, it has been seen that the first three POD modes capture almost $99.96 \%$ of energy which implies that these three POD modes are more than sufficient to represent the dynamics of the beam structure. 


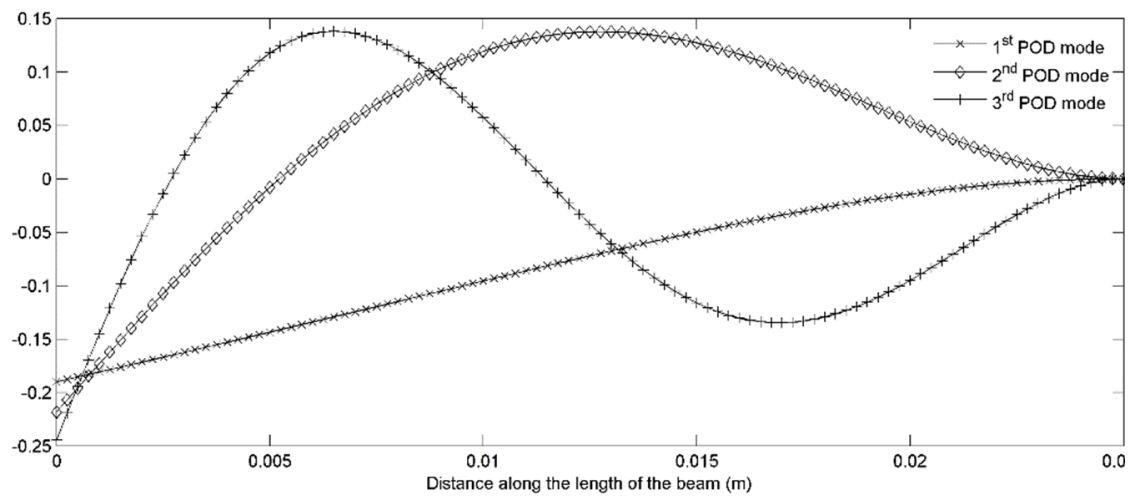

Figure 4: POD mode shapes of the beam structure.

\subsubsection{Governing equation}

The physical governing system for the structure is given by the Euler-Bernoulli's beam equation as per eqn (5), here damping has not been considered.

$$
E I \frac{\partial^{4} y(x, t)}{\partial x^{4}}+\rho A \frac{\partial^{2} y(x, t)}{\partial t^{2}}=f(x, t)
$$

where $\mathrm{E}$ is the Young's modulus, I is the moment of inertia of the cross section of the beam, $\rho$ is the density of the beam, A is the cross section area of the beam, $y(x, t)$ is the transverse deflection, $f(x, t)$ is the applied force on the beam.

The displacement field $y(x, t)$ is represented as the summation of multiplication of temporal variable and spatial variable. By projecting eqn (5) onto global basis functions using Galerkin projection with time varying coefficients $\beta_{i}(t)$ and spatial varying function $b_{i}(x)$ as in eqn (6).

$$
\hat{y}(x, t)=\sum_{i=1}^{m} \beta_{i}(t) b_{i}(x)
$$

As we know already the columns of the $\mathrm{U}$ (refer eqn (4)) represents global basis functions $b_{i}(x)$ for the displacement field. And as we have already seen in section 4.2.1 that the three POD global basis are more than sufficient to capture the dynamics of the beam, hence $m=3$ in eqn (6).

Galerkin projection is used to reduce the order and obtain the ODEs using governing partial differential eqn (5), which requires the residual of the PDEs to be orthogonal to each basis function. The Galerkin projection of eqn (5) using approximation as $\hat{y}(x, t)$ from eqn (6), results in the following ODE eqn (7).

$$
M \ddot{\beta}+K \beta=f
$$

where 
mass matrix, $M_{i j}=\int_{\mathrm{L}} \rho A b_{i} b_{j} d x$

stiffness matrix, $K_{i j}=\int_{\mathrm{L}} E I \frac{\partial^{2} b_{i}}{\partial x^{2}} \frac{\partial^{2} b_{j}}{\partial x^{2}} d x$

force matrix, $f_{i}=\int_{L} b_{i} f(x, t) d x$

where $\mathrm{L}$ indicates the integration over the length of the beam.

\subsection{Fluid structure interface using one-way coupling}

For the one-way coupling we replace the forcing term of the governing ODE of the beam in eqn (7) with the pressure forcing term of the fluid domain $(\delta p)$ from eqn (3), so the new coupled governing equation becomes as eqn (8) as follows:

where

$$
M \ddot{\beta}+K \beta=f_{p}
$$

$$
f_{p_{i}}=\int_{L} b_{i} \delta p(x, t) *(\text { width }) d x
$$

\section{Results and conclusions}

Using eqn (9) $f_{p_{i}}$ can be pre-calculated for each time instant for each POD mode. Here, it is for just first three POD modes. Then, ODE eqn (8) has been solved for each discretised time step using ODE45 function in Matlab software. The results are validated using coupled FSI simulation using Abaqus Co-simulation and the obtained results are compared in fig. 5 for the displacement at the free end of the cantilevered beam.

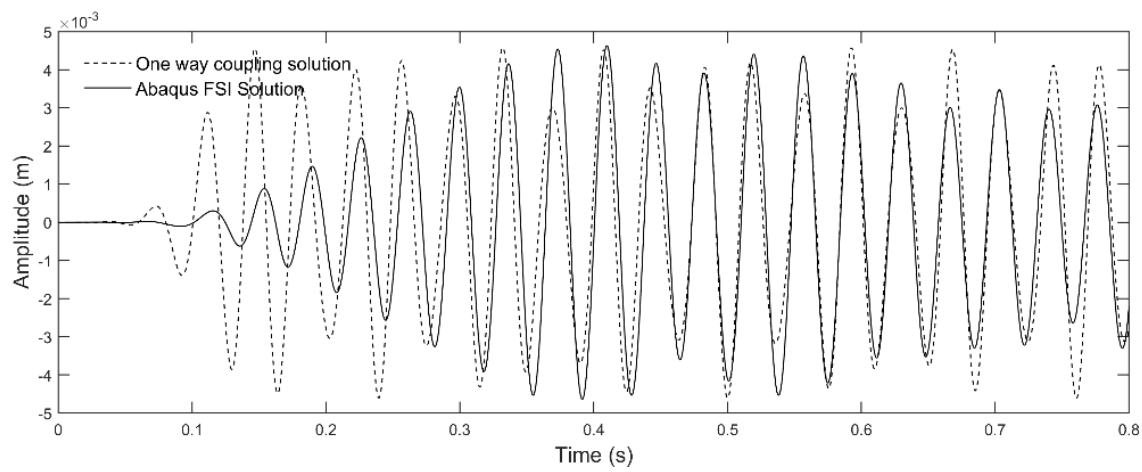

Figure 5: Comparison of the results for one-way coupling using POD-ROM and Abaqus FSI simulation results for the free end of the cantilever beam.

From fig. 5, it has been observed that the frequency of the vibration of the beam matches well in both the cases and also it provides a good estimation of the amplitude of vibration of the beam without going for computationally costly coupled dynamic simulation. The characteristic of a dynamic of the system can be found by taking the Fast Fourier Transform (FFT) of a displacement field of the beam for FSI response and the one-way coupling response as in fig. 6, which 
shows the dynamics of the one-way coupled beam solution and fully coupled FSI simulation has good similarity.

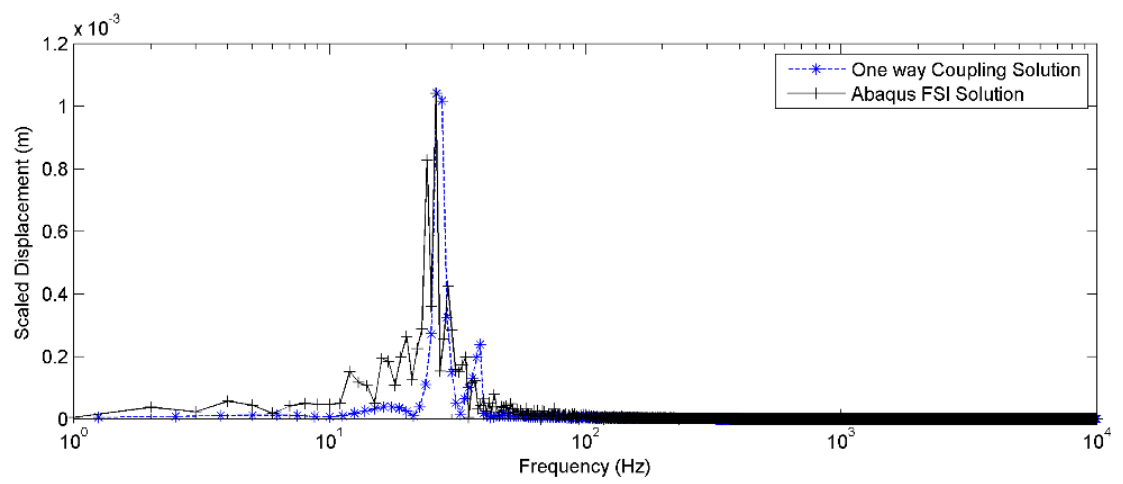

Figure 6: Comparison of FFT of one-way coupling and Abaqus FSI.

At the same time, it should be noted that, as we have neglected the effect of the motion of the beam on the fluid flow implies that the large deformation of the beam could not be predicted exactly. However, for small deformation of the beam it can be predicted pretty well. And the benefit of such one-way coupling strategy is that in the same interface boundary of the FSI domain, we can change the structural material properties and predict the results without going for a complete FSI problem.

\section{References}

[1] Elmer S. Hung \& Stephan D. Senturia, Generating Efficient Dynamical Models for Microelectromechanical Systems from a Few Finite-Element Simulation Runs, IEEE Journal of Microelectromechanical Systems, Vol. 8, No. 3, pp. 280-289, September 1999.

[2] Imran Akthar, Ali H. Nayfeh \& Calvin J. Ribbens, On the stability and extension of reduced order Galerkin models in incompressible flows, Theor. Comput. Fluid Dyn, pp. 213-237, 2009.

[3] Zongxian Liang \& Haibo Dong, Virtual Force Measurement of POD Modes for A flat Plate in Low Reynolds Number Flows, 52nd Aerospace Sciences Meeting, AIAA SciTech, 2014.

[4] A. Tallet, C. Allery, C. Leblond \& E. Liberge, A minimum residual projection to build coupled velocity-pressure POD-ROM for incompressible NavierStokes equation, Communication in Nonlinear Science and Numerical Simulation, Vol. 22, pp. 909-932, May 2015.

[5] L. A. Weinstein, M. R. Cacan, P. M. So \& P. K. Wright, Vortex shedding induced energy harvesting from piezoelectric materials in heating, ventilation and air conditioning flows, Smart Materials and Structures, Vol. 21, 2012. 
[6] Yunus A. Cengel \& John M. Cimbala, Differential analysis of fluid flow (Chapter 9). Topics in Fluid Mechaics Fundamentals and Applications, Tata McGraw Hill Education pvt. Ltd., pp. 437-499, 2014.

[7] L. Huang, Flutter of cantilevered plates in axial flow, Journal of Fluids and Structures, 9, pp. 127-147, 1995.

[8] Sudipto Banerjee \& Anindya Roy, Singular value and Jordan Decompositions (Chapter 12). Topics in Linear Algebra and matrix analysis for statistics, CRC press, pp. 371-399, 2014. 